\title{
RIVOAL (Isabelle), Les Maîtres du Secret. Ordre mondain et ordre religieux dans la communauté druze en Israël
}

Paris, Éditions de l'E.H.E.S.S., 2000, 432 p. (index, illustr., cartes, bibliogr.)

\section{Malika Zeghal}

\section{OpenEdition}

Journals

Édition électronique

URL : http://journals.openedition.org/assr/20356

DOI : $10.4000 /$ assr.20356

ISSN : $1777-5825$

Éditeur

Éditions de l'EHESS

Édition imprimée

Date de publication : 31 décembre 2000

Pagination : 122-124

ISBN : 2-222-96698-1

ISSN : 0335-5985

\section{Référence électronique}

Malika Zeghal, «RIVOAL (Isabelle), Les Maitres du Secret. Ordre mondain et ordre religieux dans la communauté druze en Israël », Archives de sciences sociales des religions [En ligne], 112 I octobredécembre 2000, document 112.45, mis en ligne le 19 août 2009, consulté le 21 septembre 2020. URL : http://journals.openedition.org/assr/20356 ; DOI : https://doi.org/10.4000/assr.20356 


\section{RIVOAL (Isabelle), Les Maîtres du Secret. Ordre mondain et ordre religieux dans la communauté druze en Israël}

Paris, Éditions de l'E.H.E.S.S., 2000, 432 p. (index, illustr., cartes, bibliogr.)

\section{Malika Zeghal}

\section{RÉFÉRENCE}

RIVOAL (Isabelle), Les Maîtres du Secret. Ordre mondain et ordre religieux dans la communauté druze en Israël, Paris, Éditions de l'E.H.E.S.S., 2000, 432 p. (index, illustr., cartes, bibliogr.)

1 L'ouvrage d'I.R. n'est pas une étude sur les rapports entre la communauté druze d'Israël et l'État au sein duquel elle est intégrée. L'auteur a pris, avec bonheur, le parti de décrire l'«entre-soi » d'une communauté que définit la religion («ciment de la communauté ») et d'étudier les liens subtils, complexes, mais jamais unidimensionnels qui unissent le religieux et le politique. Elle souligne l'originalité d'une religion très faiblement ritualisée où le dogme demeure secret pour la plupart des non initiés, et montre que le religieux et le politique ne se définissent pas dans un rapport de confusion.

Une enquête ethnographique fouillée permet de redonner la parole aux habitants de deux villages druzes situés sur les hauteurs du Mont Carmel non loin de Haïfa. L'étude du politique est saisie au niveau local, dans les rivalités qui opposent les diverses factions familiales. Le religieux est, lui, saisi à travers la sphère des hommes de religion. Après avoir décrit l'histoire des deux villages (en particulier les rapports avec les musulmans palestiniens et avec l'État d'Israël après 1948), elle décrit leur vie politique, la manière dont les habitants regardent leur propre passé et réussissent (ou échouent) à construire les figures du chef, des factions et des alliances, en vue de capter le 
pouvoir. Elle montre que cet ordre du mondain, ou de la dunya, n'a que peu à voir avec celui du religieux ou du dîn.

3 Au niveau du village, explique-t-elle, les affaires mondaines ne sont pas définies par le religieux : «Il n'y a pas d'oppositions politiques internes uniquement fondées sur des critères de religion" (p.129). Sur ce plan, elle décrit une séparation de ces deux sphères. Cependant, l'entité du village n'est pas l'unité principale de référence. L'A. se réfère primordialement aux pratiques et aux discours des habitants, sans les enfermer dans la coquille compacte et a priori structurante du village. A travers leur parole, recueillie sur le terrain, la monographie apporte une vision éclairante sur une communauté qui se définit par le religieux mais ne se fonde pas exclusivement sur la religion dans le traitement de ses affaires mondaines. Si ces discours enrichissent le texte, on sait peu de choses sur les caractéristiques sociologiques des individus qui sont cités et sur les contextes spatiaux et historiques dans lesquels ils s'expriment. Ils permettent toutefois de recomposer les relations entre patrilinéages, et surtout de montrer la place centrale qu'occupent les femmes dans les enjeux qui lient entre elles les factions.

4 L'A. refuse de procéder à une analyse en termes de " crise d'identité ", soulignant que l'appartenance des Druzes à un État moderne (dans ce cas Israël) n'est pas déstabilisatrice ou productrice de situations de crise. Elle critique le réductionnisme de l'analyse politique qui mobilise l'attention sur la recherche par le groupe ethnique de son miroir politique dans un micro État : «Ces analyses d'inspiration juridico-politiques s'enferment dans une vision réductionniste du tout ou rien, de l'édification nationale ou de la crise et du conflit»(p.13). Mais plutôt que de renouveler cette analyse politique, elle préfère alors - dans un premier temps - laisser de côté l'analyse de l'État.

5 L'A. décrit la sphère des hommes de religion, en marquant des différences nettes avec les hommes de religion en islam sunnite, bien que beaucoup des traits qu'elle décrit se rapprochent nettement des pratiques et des idées de la mystique musulmane. La différenciation qu'elle propose entre shaykh temporel (l'homme politique) et shaykh religieux (l'homme de religion) est fort instructive. En poussant le raisonnement, on pourrait presque dire que le premier est za'im (chef) avant d'être Druze, même si son identité de Druze ne cesse de le définir. Le second est Druze avant tout, mais il est entièrement. spatialement comme moralement, impliqué dans la communauté. Il n'échappe pas aux structures qui sont dessinées par les lignages. Lorsque le premier dépasse la structure locale du village c'est pour se mettre en relation, par exemple, avec des instances qui restent mondaines. Lorsque le second s'insère dans une structure globale, c'est pour trouver «sa place dans une structure religieuse coiffée par une autorité spirituelle» (p. 192).

6 Cette analyse de l'interaction entre communauté religieuse, espace politique et autorité religieuse est très importante, en ce sens qu'elle révèle, dans une description novatrice, un emboîtement assez complexe de ces sphères, où aucune ne se confond avec les autres. Instructive aussi, la différence entre le monde masculin et féminin quand il s'agit d'atteindre "un au-delà des limites du village et un au-delà du monde social » (p. 192). Les hommes l'atteignent par la retraite et l'initiation, mais les femmes par le moment des funérailles.

7 L'A. décrit l'organisation en réseau et le fonctionnement des lieux de culte druzes (khalwa) et des tombeaux des saints. Elle montre que la mise en place de ces khalwas est aussi en un sens liée à des oppositions factionnelles. Elle décrit la fonction juridique de 
cette hiérarchie souple ainsi que le système des waqfs, puis l'institutionnalisation de la hiérarchie spirituelle druze.

8 Selon l'A., l'insertion des religieux Druzes dans la communauté les place à la limite du divin et du mondain, constante position à « la limite » qu'on retrouve d'ailleurs dans le groupe des hommes de religion sunnites. Les hommes de religion se trouvent au centre de la société druze sans toutefois définir ou structurer de manière monolithique la minorité druze. Ils sont les gardiens de deux frontières, celle du secret de la religion, et celle de l'honneur de la communauté, qui passe par les femmes. Même s'ils sont au centre de la communauté. il n'y a pas de juxtaposition stricte entre politique et religion. Mais aux niveaux de la khalwa, et des tombeaux, inscrits pleinement dans la communauté, le chef spirituel échappe, car il effectue un parcours différent des autres hommes de religion. C'est pourquoi l'A. décrit tout au long d'un chapitre passionnant, la généalogie, l'initiation, la vie et le passage à la sainteté du shaykh Amin Tarif qui en 1928 devint chef spirituel de la communauté. Il faudra d'abord que le shaykh s'abîme hors du monde, avant de pouvoir faire un parcours à l'envers et prendre la charge de "direction religieuse et mondaine », d'homme de Dieu et de médiateur, qui dépasse la seule communauté druze d'Israël. Et c'est dans son analyse du chef spirituel que la réflexion autour de l'État est réintroduite. C'est en effet, selon l'A., le chef spirituel qui inscrit la communauté dans l'État d'Israël.

Ce sont pourtant les femmes, qui, pour l'A., vont «tenir» la sociéte druze toute ensemble dans une unité idéale, et non l'institution religieuse. À travers les rituels funéraires, et la place que les femmes y tiennent, I.R. montre comment les femmes " dépassent, à leur manière, les limites des communautés locales pour dessiner les contours idéaux de cette unité ultime de la communauté »(p.343). Ces pratiques permettent de réaffirmer la mémoire du groupe à travers les femmes, qui sont elles aussi gardiennes du secret et qui « contrôlent » la réincarnation.

L'ouvrage, très agréable à lire, décrit très clairement le système religieux druze et introduit des comparaisons intéressantes avec d'autres systèmes religieux, notamment à travers les travaux de Peter Brown, ou de Michael Gilsenan. L'A. lance aussi de nouvelles pistes de recherche sur la transmission du texte sacré par la copie, sur le rôle du silence, le statut de l'écrit et de l'oralité. On peut s'étonner d'une définition de la sunna comme "commentaires interprétatifs de [la] parole [divine]»(p.175), ou de cette affirmation un peu rapide selon laquelle l'entrée dans une confrérie au Maroc est essentiellement un choix individuel indépendant d'une inscription sociale (p. 187).

On peut se poser la question du traitement de l'État dans ce travail. On constate sa très forte présence, que le lecteur peut parfois ressentir comme refoulée par l'A. Non pas qu'il fallait absolument traiter de sa présence et des relations entre la communauté et celui-ci. Le choix de la problématique a été annoncé en introduction, et de manière tout à fait fondée et légitime. On voit toute l'importance de l'État dans les passages historiques et dans le chapitre qui traite du chef spirituel de la communauté. Mais l'État n'est-il présent que par cet intermédiaire ? Les «habitants " des villages qui ont été étudiés ne sont sûrement pas indifférents à leur insertion dans l'État d'Israël, et cela aussi définit le "politique ». L'A. aurait pu annoncer que sa définition du "politique » restait de ce fait « restreinte ». Si, comme elle le dit, l'État est un « démiurge créateur de spécificité ethnique» (p. 386), il ne faut pas oublier que les hommes et les groupes savent aussi ré-inventer et faire fonctionner cette spécificité. Ce que d'ailleurs, elle a magistralement démontré, grâce à un travail de terrain très riche et une interprétation 
très subtile. Mais cette spécificité n'est pas plus "authentique » ou "vraie " parce qu'elle émerge du plus intime de la communauté étudiée. Peut-être l'identité d'une communauté doit-elle se définir sur un mode pluriel, au travers d'instances diverses, et non seulement dans l'« entre soi », même secret, de cette communauté. 